\title{
UNIVERSITYOF
}

FORWARD

THINKING

WESTMINSTER用

WestminsterResearch

http://www.westminster.ac.uk/westminsterresearch

Positive body image is positively associated with hedonic

(Emotional) and eudaimonic (Psychological and Social) well-

being in british adults

Swami, V., Weis, L., Barron, D. and Furnham, A.

This is an Accepted Manuscript of an article published by Taylor \& Francis in The Journal of Social Psychology, DOI: 10.1080/00224545.2017.1392278. The final definitive version is available online:

https://dx.doi.org/10.1080/00224545.2017.1392278

(C) 2017 Taylor \& Francis

The WestminsterResearch online digital archive at the University of Westminster aims to make the research output of the University available to a wider audience. Copyright and Moral Rights remain with the authors and/or copyright owners.

Whilst further distribution of specific materials from within this archive is forbidden, you may freely distribute the URL of WestminsterResearch: ((http://westminsterresearch.wmin.ac.uk/).

In case of abuse or copyright appearing without permission e-mail repository@westminster.ac.uk 


\section{Positive body image is positively associated with hedonic (Emotional) and eudaimonic (Psychological and Social) well-being in british adults}

\section{Viren Swami, Laura Weis, David Barron \& Adrian Furnham}

To cite this article: Viren Swami, Laura Weis, David Barron \& Adrian Furnham (2017):

Positive body image is positively associated with hedonic (Emotional) and eudaimonic (Psychological and Social) well-being in british adults, The Journal of Social Psychology, DOI: 10.1080/00224545.2017.1392278

To link to this article: $\mathrm{http}: / / d x . d o i . o r g / 10.1080 / 00224545.2017 .1392278$

Accepted author version posted online: 20

Oct 2017.

Submit your article to this journal $[\pi$

Џll Article views: 5

View related articles $\sqsubset$

View Crossmark data \ulcorner 


\title{
Positive Body Image is Positively Associated with Hedonic (Emotional) and Eudaimonic (Psychological and Social) Well- Being in British Adults
}

\begin{abstract}
Studies examining associations between positive body image and well-being have used a limited array of measures of each construct. To rectify this, we asked an online sample of 1148 UK adults to complete a range of measures of positive body image (body appreciation, body image flexibility, body pride, body acceptance from others) and a multi-dimensional measure of well-being (emotional, psychological, and social). Results showed that, once the effects of age and body mass index (BMI) had been accounted for, body appreciation significantly predicted all dimensions of well-being. Other positive body image measures emerged as significant predictors, but patterns of associations were mixed across sex and well-being dimension. Additional analyses showed that women had significantly lower scores than men on most body image measures, and that BMI was negatively associated with all body image measures. These results have implications for the promotion of well-being, which we discuss.
\end{abstract}

Keywords: Positive body image; Well-being; Body appreciation; Hedonic wellbeing; Eudaimonic well-being 
Over the past decade, scholars have turned their attention to the construct of positive body image, signalling an important shift in the field from a focus on pathology to a more holistic and comprehensive account of the body image concept (for reviews, see Tylka, 2011, 2012; Tylka \& Wood-Barcalow, 2015a). Broadly speaking, positive body image can be defined as love, respect, and acceptance of one's body (Tylka \& Wood-Barcalow, 2015a; Wood-Barcalow, Tylka, \& Augustus-Horvath, 2010). Core features of positive body image include an appreciation of the uniqueness of one's body, a compassionate acceptance of the body including those aspects that are inconsistent with societally-prescribed ideals, an emphasis on the body's functionality rather than aesthetics, and a body-protective outlook in which positive information is internalised and negative information is rejected or reframed (Tylka, 2011, 2012; Tylka \& Wood-Barcalow, 2015a).

Importantly, accumulating evidence suggests that positive and negative body image are independent constructs (for a review, see Tylka \& Wood-Barcalow, 2015a). That is, positive body image does not fall on the same continuum as, nor is it the polar opposite of, negative body image (Avalos, Tylka, \& Wood-Barcalow, 2005; Tylka \& Wood-Barcalow, 2015a). This is noteworthy because positive and negative body image may display unique relationships with outcome variables, such as health-related behaviours and well-being (Andrew, Tiggemann, \& Clark, 2016; Gillen, 2015; Halliwell, 2015). In particular, positive body image may have a direct positive impact on outcome variables or may have an impact on outcome variables through unique, indirect pathways (e.g., avoiding damaging societal influences). Intervention strategies that are attuned to both positive and negative body image, therefore, offer a more holistic approach for optimising health and well-being (Cook-Cottone, Tribole, \& Tylka, 2013).

In terms of mental health specifically, there is now a wealth of evidence indicating that positive body image is directly associated with improved outcomes. For example, studies 
have consistently reported that positive body image is positively associated with a range of indicators of well-being, including optimism, positive affect, self-compassion, life satisfaction, and subjective happiness (Alleva, Martijn, Veldhuis, \& Tylka, 2016; Avalos et al., 2005; Dalley \& Vidal, 2013; Marta-Simões, Ferreira, \& Mendes, 2016; Raque-Bogdan, Piontkowski, Hui, Ziemer, \& Garriot, 2016; Swami \& Ng, 2015; Swami, Ng, \& Barron, 2016; Swami, Tran, Stieger, Voracek, \& The YouBeauty.com Team, 2014; Tylka \& Kroon van Diest, 2013; Wasylkiw, MacKinnon, \& MacLellan, 2012). In addition, positive body image may translate into positive feelings toward the self as a whole: a series of studies have consistently reported that positive body image is associated with higher self-esteem (Gillen, 2015; Ng, Barron, \& Swami, 2015; Swami, Airs, Chouhan, Padilla Leon, \& Towell, 2009; Swami, Barron, Weis, \& Furnham, 2016; Swami, Henry, Peacock, Roberts-Dunn, \& Porter, 2013; Swami \& Ng, 2015; Swami, Ng, et al., 2016; Swami, Stieger, Haubner, \& Voracek, 2008; Swami, von Nordheim, \& Barron, 2016; Tylka \& Kroon van Diest, 2013; Tylka \& Wood-Barcalow, 2015b).

While this body of evidence appears conclusive, two issues currently limit our understanding of the associations between positive body image and well-being. First, although positive body image is a multi-faceted construct, consisting of multiple dimensions that demonstrate minimal conceptual overlap (for reviews, see Tylka \& Wood-Barcalow, 2015a; Webb, Wood-Barcalow, \& Tylka, 2015), the vast majority of studies have typically focused on singular facets. In particular, most of the extant literature has focused on the facet of body appreciation (i.e., favourable opinions of one's body, body acceptance, bodily respect, and a protective cognitive style that rejects unrealistic appearance ideals; Avalos et al., 2005; Tylka \& Wood-Barcalow, 2015b), to the exclusion of other facets of positive body image. Therefore, existing studies may be neglecting unique associations between multiple facets of positive body image and indices of well-being. 
Beyond body appreciation, few studies have examined associations between facets of positive body image and well-being, although the available evidence does suggest positive relationships (for a review, see Webb et al., 2015). For example, there is some evidence of positive associations between body image flexibility (i.e., a tendency to compassionately embrace, rather than avoid, aversive body-related thoughts and feelings) and self-compassion and self-esteem, respectively (Sandoz, Wilson, Merwin, \& Kellum, 2013). Likewise, body pride (i.e., strong and positive affect towards the body) has been found to be positively associated with self-esteem (Castonguay, Gilchrist, Mack, \& Sabiston, 2013). Taken together, these studies point toward positive associations between facets of positive body image and indices of well-being, but previous studies have not considered these multiple facets within the same piece of research.

In addition, a second issue needs also to be considered. Specifically, the studies reviewed above have focused on indices of hedonic well-being, which equates mental health with avowed happiness in life, positive self-regard, or the experience of positive emotions (that is, well-being is primarily operationalised in terms of emotional well-being). Whilst this focus is warranted, some scholars believe it misses other key facets of subjective well-being (for a review, see Keyes, Shmotkin, \& Ryff, 2002). Thus, in contrast to hedonic happiness, eudaimonic well-being equates happiness with human potential that, if realised, results in positive person functioning (Keyes et al., 2002). In this view, eudaimonic happiness consists of facets relating to psychological well-being (i.e., the challenges that individuals face as they strive to realise their unique talents; Ryff, 1989; Ryff \& Keyes, 1995) and social well-being (i.e., the degree to which individuals are functioning well in the social realm; Keyes, 1998).

This holistic approach to well-being adequately distinguishes between the experience of positive well-being and the absence of symptoms of mental ill-health (Keyes, 2002, 2005). More importantly, factor analytic studies have supported a three-dimensional structure of 
well-being, consisting of emotional, psychological, and social facets (e.g., Doré, O’Loughlin, Sabiston, \& Fournier, 2016; Keyes, 2005; Keyes et al., 2008; Lamers, Westerhof, Bohlmeijer, ten Klooster, \& Keyes, 2010; Petrillo, Capone, Caso, \& Keyes, 2015; Westerhof \& Keyes, 2010). That is, these facets of well-being can be considered to be discrete aspects that, when taken together, provide a more global index of the degree to which an individual is flourishing in life (Keyes, 2002). Therefore, a consideration of the multi-dimensional nature of well-being provides the fullest account of positive mental health (Westerhof \& Keyes, 2010).

Applied to existing studies of positive body image, it might be argued that scholars have heretofore only examined associations with a narrow index of well-being (i.e., emotional), neglecting additional and important facets (i.e., psychological and social) that would provide a more holistic accounting of well-being. Aside from this neglect, there are additional reasons why examining relationships between body image and broader facets of well-being are important. First, it would be useful to examine whether having positive body image is related one's striving to become a better person and to realise one's potential. If such a relationship can be established, it would highlight the importance of positive body image in terms of individual fulfilment. Second, establishing a relationship between positive body image and social well-being would highlight the extent to which body image is associated with an individual's optional functioning in society. In short, there is scope for more fully examining the extent to which positive body image is associated with being mentally healthy. 


\section{The Present Study}

Here, we sought to add to extant knowledge by examining associations between multiple facets of positive body image and multi-dimensional aspects of well-being. In terms of well-being, we included a measure that taps all three aspects of the multi-dimensional model of well-being, thus providing greater coverage of the construct than previous studies. In terms of body image, we included a measure of body appreciation, given that this is the dominant way in which positive body image is currently operationalised (Webb et al., 2015). To this, we added measures of body image flexibility and body pride in order to provide broader coverage of the positive body image construct. In addition, because we were interested in social outcomes, we also included a measure of body acceptance by others (i.e., an individual's perceived acceptance of their body from external sources). We hypothesised that facets of positive body image would be significantly and positively associated with emotional, psychological, and social well-being, respectively.

We investigated these issues in a large, online sample of British adults, which allowed us to interrogate additional aspects of the literature. First, we were able to examine sex differences in positive body image. Previous studies have typically reported that men have significantly more positive body appreciation and body image flexibility than women (see Tiggemann, 2015; Webb et al., 2015), possibly because men have greater access to societal and individual resources that promote positive body image (Swami, Stieger et al., 2008). However, it is important to note that effect sizes have been small-to-moderate at best and null findings have previously been reported in British adults in terms of body appreciation (Swami, Hadji-Michael, \& Furnham, 2008). Likewise, a study of Canadian adults reported no significant sex difference in body pride (Pila, Brunet, Crocker, Kowalski, \& Sabiston, 2016), 
but to our knowledge sex differences in body acceptance from others have not been previously investigated.

Second, we were also able to examine associations between facets of body image and respondent age. Studies of the relationship between age and body appreciation (e.g., Swami et al., 2014; Tiggemann \& McCourt, 2013) and body image flexibility (Ferreira, PintoGouveia, \& Duarte, 2011) have suggested positive, albeit small-to-moderate, associations. Associations between body pride and age have not been previous examined, whereas cohort data reported by Augustus-Horvath and Tylka (2011) suggests that body acceptance by others may decline with increasing age. Third, we examined associations between positive body image and self-reported body mass index (BMI). The evidence base here appears to be more robust, suggesting an inverse relationship between BMI and facets of positive body image (see Webb et al., 2015), although again the strength of report associations has been variable.

\section{Method}

\section{Participants}

Our sample consisted of 716 women and 432 men, all of whom were British citizens. Participants ranged in age from 18 to 81 years $(M=34.87, S D=12.08)$ and in self-reported BMI from 13.63 to $48.05 \mathrm{~kg} / \mathrm{m}^{2}(M=25.95, S D=5.67)$. Most participants self-reported their ethnicity as being British White (88.0\%), while 6.3\% were British Asian, 2.3\% were Black or African Caribbean, and 3.5\% were of some other ancestry. The majority of participants selfreported their sexual orientation as being heterosexual $(87.5 \%)$, while in terms of relationship status $31.6 \%$ were single, $10.1 \%$ were partnered but not cohabiting, $23.3 \%$ were partnered and cohabiting, 29.2\% were married and cohabiting, and the remainder were of some other status. In terms of educational qualifications, $26.5 \%$ had completed minimum secondary schooling, 38.4\% had an undergraduate degree, $21.8 \%$ had a postgraduate degree, $7.5 \%$ were still in full-time education, and 5.8\% had some other qualification. 


\section{Measures}

Body appreciation. Participants were asked to complete the Body Appreciation Scale-2 (BAS-2; Tylka \& Wood-Barcalow, 2015b). This is a 10-item scale that assesses acceptance of one's body, respect and care for one's body, and protection of one's body from unrealistic beauty standards (sample item: "I respect my body"). All items were rated on a 5point scale, ranging from 1 (Never) to 5 (Always), and an overall score was computed as the mean of all items. Higher scores on this scale reflect greater body appreciation. BAS-2 scores have been shown to have a one-dimensional factor structure, as well as being judged adequate in terms of internal consistency estimates, test-retest reliability after three weeks, and indices of convergent and discriminant validity, in college and community samples of U.S. adults (Tylka \& Wood-Barcalow, 2015b). In the present study, Cronbach's $\alpha$ for this scale was .95 in women and .93 in men.

Body acceptance. Participants were also asked to complete the 10-item Body Acceptance by Others Scale (BAOS; Avalos \& Tylka, 2006). This is a measure of an individual's perceptions of acceptance for, and receiving messages reflecting acceptance of, their body shape and weight from friends, family, dating partners, society, and the media (sample item: "I've felt acceptance from my friends regarding my body shape and/or weight"). Participants rated the frequency of these experiences using a 5-point scale, ranging from 1 (Never) to 5 (Always). An overall score was computed as the mean of all items, so that higher scores reflect greater perceived body acceptance from others. In U.S. adults, BAOS scores have been found to have a one-dimensional factor structure, adequate test-retest reliability after three weeks, and adequate patterns of construct validity (Avalos \& Tylka, 2006). Here, Cronbach's $\alpha$ for this scale was .90 in women and .92 in men.

Body pride. To measure body pride, we used the Authentic Pride subscale of the Body and Appearance Self-Conscious Emotions Scale (BASES-AP; Castonguay et al., 2014). 
This 6-item subscale measures body pride as a sense of personal appearance-related achievement (sample item: "I am proud of my appearance efforts"). Items were rated on a 5point scale, ranging from 1 (Never) to 5 (Always), and scores were averaged so that higher scores reflect greater authentic body pride. Data drawn from North American adults supports the factor structure of the BASES, and estimates supported the internal consistency, testretest reliability after two weeks, and validity of the BASES subscales (Castonguay et al., 2014). Here, Cronbach's $\alpha$ for this subscale was .95 in both women and men.

Body image flexibility. To measure body image flexibility, we used the 12-item Body Image-Acceptance and Action Questionnaire (BI-AAQ; Sandoz et al., 2013). This scale measures the degree of negative-body related thoughts, behaviours, and affect that stifle growth when experiencing aversive body-related thoughts and feelings (sample item: "I care too much about my weight and body shape"). Webb et al. (2015) have suggested that this measure provides a useful, if preliminary, measure of body image flexibility. Items were rated on a 7-point scale, ranging from 1 (Never true) to 7 (Always true). An overall score for the BI-AAQ was computed as the mean of all reverse-coded items, so that higher scores reflect greater body image flexibility (Sandoz et al., 2013). In U.S. adults, BI-AAQ scores have been shown to have a one-dimensional factor structure, adequate internal consistency, adequate test-retest reliability up to three weeks, and adequate patterns of construct validity (Sandoz et al., 2013). In the present study, Cronbach's $\alpha$ for this scale was .95 in women and men.

Well-being. Participants were asked to complete the Short Form of the Mental Health Continuum (MHC-SF; Keyes, 2005). The 14 items of this measure were selected as the most prototypical items representing well-being from the Long Form, 40-item version of the scale (Keyes, 2005, 2006). In addition, we opted for the Short Form, rather than Long Form, of this measure because the former is the more widely-used, provides a clearer standard for the 
assessment of positive mental health, and does not suffer from concerns over the internal reliability that affect the Long Form (Keyes, 2007). Three items on the MHC-SF tap emotional well-being (sample item: "During the past month, how often did you feel happy?", six items tap the dimensions of Ryff's (1989) model of psychological well-being (sample item: "During the past month, how often did you feel that you liked most parts of your personality?", and five items tap the dimensions of Keyes' (1998) model of social well-being (sample item: "During the past month, how often did you feel that you had something important to contribute to society?"). Items were rated on a 6-point scale ranging from 1 (Never) to 6 (Every day) and subscale scores were computed as the mean of items associated with each dimension. Higher scores on each subscale reflect greater well-being. The MHCSF's three-dimensional factor structure has been confirmed in nationally representative samples of U.S. adults (Gallagher, Lopez, \& Preacher, 2009). In addition, scores on the scale have been found to have adequate internal consistency estimates, adequate test-retest reliability up to nine months, and adequate patterns of discriminant validity (Lamer et al., 2010). In the present study, Cronbach's $\alpha$ for emotional well-being was .90 for women and men, for psychological well-being was .85 for women and .88 for men, and for social wellbeing was .87 for women and .88 for men.

Body mass index. Participants self-reported their height and weight on open-ended items. We standardised this data so as to present height in metres and weight in kilogrammes, and self-reported BMI was subsequently computed as $\mathrm{kg} / \mathrm{m}^{2}$. Where computations resulted in improbable BMI values $\left(<12\right.$ or $\left.>50 \mathrm{~kg} / \mathrm{m}^{2}\right)$, we replaced these using the mean replacement method $(<4.0 \%$ of the total dataset). Self-reported height and weight data are strongly correlated with measured data and can be reliably used in population studies (Spencer, Appleby, Davey, \& Key, 2002). 
Demographics. We asked participants to report demographic data consisting of sex, age, ethnicity, sexual orientation, relationship status, and highest educational qualification. Procedure

The project was approved by the relevant university ethics committee (approval number: ETH1617-0014). Data were collected via the Prolific Academic website, a crowdsourcing Internet marketplace that allows individuals to complete academic surveys for monetary compensation, between November $8^{\text {th }}$ and $11^{\text {th }}, 2016$. Crowdsourcing Internet marketplaces are increasingly used in body image research and have been shown to produce reliable data on perceptual and attitudinal body image as compared with offline samples (Gardner, Brown, \& Boice, 2012). The project was advertised as a study on "health, wellbeing, and personality" and included an estimated duration (10 minutes). Participation was limited to U.K. citizens of adult age, so as to achieve a relatively homogeneous sample in terms of cultural and national identity. After providing informed consent, participants were directed to the measures described above, which were presented in an anonymous form. The order of presentation of each of the scales above was counter-balanced for each participant, although the request for demographic details always appeared last. The survey also included eight items measuring conspiracist ideation, which are not analysed here. In exchange for completing the survey, participants were paid $£ 1.00$ and all participants received debriefing information at the end of the survey.

\section{Data Availability}

Our data are available at https://figshare.com/s/ff8b34b877a85f5bbc7c (doi:10.6084/m9.figshare.4239557). 


\section{Results}

\section{Sex Differences}

We first computed a series of independent-samples $t$-tests to examine sex differences on all key variables. Because of the large number of comparisons $(k)$, a Bonferroni adjustment was applied to reduce the chance of Type I error, such that $p=(1-\alpha)^{k} \approx 1-k \alpha=$ $\alpha / k=.006$ (Bland \& Altman, 1995). As can be seen in Table 1, women were significantly older than men and had significantly higher BMIs, although effect sizes were small by Cohen's (1977) standards. In addition, women had significantly lower scores than men on body appreciation, body pride, and body image flexibility, with effect sizes being small for the former two variables and medium for the latter variable. Women also had significantly lower social well-being scores than men, although the effect size of the difference was again small. There were no sex differences on body acceptance from others, emotional well-being, and psychological well-being. Because of the general pattern of sex differences, all further analyses were conducted separately for women and men.

\section{Inter-Scale Correlations}

We computed inter-scale correlations between all variables for women and men separately. As can be seen in Table 2, all four measures of body image were significantly and positively correlated with all three measures of well-being in women. In men, scores for body appreciation, body pride, and body acceptance from others were significantly and positively correlated with all three indices of well-being. Men's scores for body image flexibility were significantly and positively correlated with emotional and psychological well-being, but not social well-being. In addition, all four positive body image measures were significantly and negatively correlated with BMI in both women and men. Relationships between positive 
body image and age in women and men were more mixed, with not all associations reaching significance.

\section{Multiple Regressions}

Finally, we conducted multiple regression analyses for women and men separately. Three sets of regressions were computed with emotional, psychological, and social wellbeing, respectively, entered as the criterion variables and the body image variables, age, and BMI entered simultaneously as predictor variables. Full regression coefficients are reported in Table 3 for women and in Table 4 for men, and here we briefly summarise the main findings. Variance inflation factors (VIFs) under 10 are indicative of inconsequential colinearity (Hair, Anderson, Tatham, \& Black, 1995) and in our study all VIFs were $\leq 2.92$.

Women. The regression with emotional well-being was significant, $F(6,715)=$ $74.31, p<.001, \mathrm{Adj} . R^{2}=.38$, with body appreciation, body image flexibility, and BMI emerging as significant predictors. The regression with psychological well-being was also significant, $F(6,715)=79.74, p<.001$, Adj. $R^{2}=.40$, with body appreciation, BMI, body pride, age, and body acceptance from others all emerging as significant predictors. The final regression with social well-being was significant, $F(6,715)=43.98, p<.001, \mathrm{Adj} . R^{2}=.27$, with body appreciation, BMI, body pride, and age being significant predictors.

Men. In men, the regression with emotional well-being was significant, $F(6,431)=$ $52.46, p<.001$, Adj. $R^{2}=.42$, with body appreciation, body image flexibility, and BMI emerging as significant predictors. The regression with psychological well-being was likewise significant, $F(6,431)=49.10, p<.001$, Adj. $R^{2}=40$, with body appreciation, body acceptance from others, age, and BMI being significant predictors. Finally, the regression with social well-being was significant, $F(6,431)=32.32, p<.001$, Adj. $R^{2}=.30$, with body appreciation, body acceptance from others, and BMI emerging as the only significant predictors. 


\section{Discussion}

In the present study, we examined associations between multiple facets of positive body image and indices of hedonic and eudaimonic well-being. Our results showed that positive body image was significantly and positively associated with all measures of well-being. Indeed, of the variables included in our study, body appreciation consistently emerged as the strongest predictor of all three indices of well-being. In broad outline, these results are consistent with previous work showing that body appreciation is positively associated with indices of emotional well-being, such as positive affect, optimism, life satisfaction, and subjective happiness (Alleva et al., 2016; Avalos et al., 2005; Dalley \& Vidal, 2013; MartaSimões et al., 2016; Raque0Bogdan et al., 2016; Swami \& Ng, 2015; Swami, Ng, et al., 2016; Swami, Tran et al., 2014; Tylka \& Kroon van Diest, 2013; Wasylkiw et al., 2012).

However, as we suggested earlier, previous studies have focused on aspects of hedonic well-being, but have neglected psychological and social functioning in the measurement of positive mental health. Thus, while our results are consistent with previous work showing that positive body image is positively associated with the presence of positive emotions and overall satisfaction with life (i.e., emotional well-being), it is also important to note that positive body image was significantly and positively associated with psychological well-being (i.e., autonomy and a sense of personal growth) and social well-being (i.e., how well an individual functions in their social life as a member of a large society). In short, our results suggest that positive body image matters in terms of both hedonic and eudaimonic well-being.

One reason why this is important is because it suggests that positive body image could be leveraged in interventions aimed at promoting broad aspects of well-being. Specifically, Keyes $(2005,2006)$ suggests that individuals who exhibit high levels of hedonic and eudaimonic well-being can be considered to be flourishing (as opposed to languishing) in life. 
In term, flourishing is associated with lower incidence of mental ill-health, healthier psychological functioning, better health outcomes, and improved health care utilisation (Keyes, 2005, 2006). Our results suggest that a focus on positive body image may be one way of promoting a greater degree of flourishing in population-based studies, which may in turn bring a range of other positive health-related outcomes. That is, intervention strategies that promote positive body image may be expected to also lead to improvements in multiple indices of well-being. Having said that, our results also need to be unpacked further. In both women and men, body appreciation was consistently the strongest predictor of all three indices of well-being. It might, therefore, be suggested that a focus on improving body appreciation specifically may be warranted, not only for promoting healthier body image but also to promote improved well-being.

Other facets of positive body image showed weaker and more equivocal relationships with well-being. One conclusion that might be drawn based on these data is that, with the exception of body appreciation, different facets of positive body image show variable relationships with facets of well-being; the nature of these relationships may also vary depending on respondent sex. A further issue of note is that respondent BMI emerged as a significant predictor of all three facets of well-being for women and men. This finding is consistent with previous work showing that higher BMI is negatively associated with quality of life and emotional well-being (e.g., de Wit et al., 2010; Fontaine \& Barofsky, 2001; Sullivan, Ghushchyan, Wyatt, Wu, \& Hill, 2007; Williams, Mesidor, Winters, Dubbert, \& Wyatt, 2015). It has been suggested that higher BMIs bring health and functional capacity problems, which in turn have a detrimental effect on well-being (Böckerman, Johansson, Saarni, \& Saarni, 2014). Higher BMI may also be associated with the perceived and actual experience of discrimination, which in turn has a detrimental effect on well-being. 
In terms of positive body image specifically, the pattern of correlations with BMI were generally consistent with previous work but those with age were more equivocal. In the first instance, respondent BMI was negatively associated with all four indices of positive well-being in both women and men. This is broadly consistent with previous work (see Webb et al., 2015), although it should be noted that the strengths of the correlations were weak. In contrast, body appreciation and body pride were not significantly correlated with age in the present study, while body image flexibility was positively correlated and body image acceptance was negatively correlated with age. Even in terms of the significant correlations, however, the strengths of the associations were weak. Given similarly weak (though sometimes moderate) correlations in previous studies (Ferreira et al., 2011; Swami et al., 2014; Tiggemann \& McCourt, 2013), we would suggest that age may not be an especially strong correlate of positive body image. Finally, the significant differences between women and men in terms of positive body image were all in the expected direction, the one exception being body acceptance from others where we found no significant sex difference.

Another issue worth commenting on is the conceptual overlap $\left(r^{2}\right)$ between the four body image variables that were included in the present study. In both women and men, the largest conceptual overlap occurred between body appreciation and body pride $(50 \%$ in women, $44 \%$ in men). Body appreciation also overlapped about $26 \%$ with body acceptance from others ( $28 \%$ in women, $24 \%$ in men), and $42 \%$ with body image flexibility in women (the comparable overlap in men was only $9 \%$ ). The remaining variables shared no more than $20 \%$ of conceptual space. These results are consistent with previous work demonstrating a high degree of differentiation between facets of positive body image in women (particularly comparing body appreciation with other indices of positive body image; Webb, ButlerAjibade, \& Robinson, 2014; see also Homan \& Tylka, 2014) and lend credence to the view that positive body image is multi-faceted (Tylka \& Wood-Barcalow, 2015a). 
Strengths of the present study include the relatively large sample and measurement of multiple facets of positive body image and well-being, respectively. However, limitations of this study include the reliance on cross-sectional data, which limits our ability to draw causal inferences. Although we have interpreted our data in line with current theorising (i.e., that body image is an antecedent of well-being), it is also possible that higher well-being leads to more positive body image. Another limitation of the present study was the reliance on an online sample, which may limit the generalizability of our findings (e.g., our sample may have been over-represented in terms of higher education compared with the general population). Importantly, our subsample sizes are suggestive of a recruitment bias toward women, which is a known issue with online recruitment (e.g., Mason \& Suri, 2012). A related issue concerns possible neglected sample factors: in the U.K., for example, there are known to be ethnic differences in positive body image (Swami et al., 2009), but we were unable to interrogate this issue given the relatively small sub-sample sizes of non-White participants. In addition, some participants returned data with improbable BMI values. Although these participants accounted for $<4.0 \%$ of the total dataset and were therefore unlikely to have had a major effect on our results, future online studies could prevent this by adding a pop-up warnings in cases of unrealistic combinations of height and weight data.

A further limitation of the present study was the fact that we utilised a limited set of measures to operationalise the positive body image construct. Webb et al. (2015) have reviewed the range of measures that potentially measure discrete aspects of positive body image and it would be useful, in future work, to include a broader range of such measures. As examples, such studies could include measures of body functionality (see Abbott \& Barber, 2010; Augustus-Horvath \& Tylka, 2011; Avalos \& Tylka, 2006), body attunement and responsiveness (Daubenmier, 2005), and broad conceptualisation of beauty (Tylka \& Iannantuono, 2016). This issue raises additional questions about shared conceptual space 
between different measures of positive body image. Although the results of the present study suggest that the difference measures were relatively discrete, this is an issue that warrants further and sustained investigation. The different response scales used by different attitudinal measures of positive body image currently hampers a fuller investigation of this issue, but in future work it would be helpful to investigate the extent to which the different measures that are being used either share latent conceptual space or load onto higher-order dimensions.

In conclusion, we are in agreement with Keyes' $(2002,2005,2006)$ suggestion that including measures of both hedonic and eudaimonic well-being provides improved coverage of positive mental health. From a practical point-of-view, this is valuable because our data suggest that positive body image is not only associated with higher emotional well-being, but that it may also be associated with greater likelihood of achieving a flourishing state of being. For policy-makers and practitioners, therefore, a focus on positive body image may be one way of promoting better well-being and helping populations to flourish. Of course, it remains important to more fully understand the mechanism by positive body image exerts an influence of well-being. The most direct route to such understanding would be through longitudinal studies that include a more comprehensive array of variables. Multiple indices of positive body image should be considered for inclusion in such studies to better predict wellbeing.

\section{References}

Abbott, B. D., \& Barber, B. (2010). Embodied image: Gender differences in functional and aesthetic body image among Australian adolescents. Body Image, 7, 22-31. doi:10.1016/j.bodyim.2009.10.004

Alleja, J. M., Martijn, C., Veldhuis, J., \& Tylka, T. L. (2016). A Dutch translation and validation of the Body Appreciation Scale-2: An investigation with female university students in the Netherlands. Body Image, 19, 44-48. doi:10.1016/j.bodyim.2016.08.008 
Andrew, R., Tiggemann, M., \& Clark, L. (2016). Positive body image and young women's health: Implications for sun protection, cancer screening, weight loss, and alcohol consumption behaviours. Journal of Health Psychology, 21, 28-39. doi:10.1177/1359105314520814

Augustus-Horvath, C. L., \& Tylka, T. L. (2011). The acceptance model of intuitive eating: A comparison of women in emerging adulthood, early adulthood, and middle adulthood. Journal of Counseling Psychology, 58, 110-125. doi:10.1037/a0022129

Avalos, L. C., \& Tylka, T. L. (2006). Exploring a model of intuitive eating with college women. Journal of Counseling Psychology, 53, 486-497. doi:10.1037/00221067.53.4.486

Avalos, L. C., Tylka, T. L., \& Wood-Barcalow, N. (2005). The Body Appreciation Scale: Development and psychometric evaluation. Body Image, 2, 285-297. doi:10.1016/j.bodyim.2005.06.002

Bland, J. M., \& Altman, D. G. (1995). Multiple significance tests: The Bonferroni method. British Medical Journal, 310, 170. doi:10.1136/bmj.310.6973.170

Böckerman, P., Johansson, E., Saarni, S. I., \& Saarni, S. E. (2014). The negative association of obesity with subjective well-being: Is it all about health? Journal of Happiness Studies, 15, 857-867. doi:10.1007/s10902-013-9453-8

Castonguay, A. L., Sabiston, C. M., Crocker, P. R. E., \& Mack, D. E. (2014). Development and validation of the Body and Appearance Self-Conscious Emotions Scale (BASES). Body Image, 11, 126-136. doi:10.1016/j.bodyim.2013.12.006

Cohen, J. (1977). Statistical power analysis for the behavioral sciences. New York, NY: Routledge. 
Cook-Cottone, C. P., Tribole, E., \& Tylka, T. L. (2013). Healthy eating in schools: Evidencebased interventions to help kids thrive. Washington, DC: American Psychological Association. doi:10.1037/14180-000

Dalley, S. E., \& Vidal, J. (2013). Optimism and positive body image in women: The mediating role of the feared fat self. Personality and Individual Differences, 55, 465468. doi:10.1016/j.paid.2013.04.006

Daubenmier, J. J. (2005). The relationship of yoga, body awareness, and body responsiveness to self-objectification and disordered eating. Psychology of Women Quarterly, 29, 207219. doi:10.1111/j.1471-6402.2005.00183.x

de Wit, L., Luppino, F., van Straten, A., Penninx, B., Zitman, F., \& Cuijpers, P. (2010). Depression and obesity: A meta-analysis of community-based studies. Psychiatry Research, 178, 230-235. doi:10.1016/j.psychres.2009.04.015.

Doré, I., O’Loughlin, J. O., Sabiston, C. M., \& Fournier, L. (2016). Psychometric evaluation of the Mental Health Continuum-Short Form (MHC-SF) in French Canadian young adults. Canadian Journal of Psychiatry, in press. doi:10.1177/0706743716675855

Ferreira, C., Pinto-Gouveia, J., \& Duarte, C. (2011). The validation of the Body Image Acceptance and Action Questionnaire: Exploring the moderator effect of acceptance on disordered eating. International Journal of Psychology and Psychological Therapy, 11, $327-345$

Fontaine, K. R., \& Barofsky, I. (2001). Obesity and health-related quality of life. Obesity Reviews, 2, 173-182. doi:10.1046/j.1467-789x.2001.00032.x

Gallagher, M. W., Lopez, S. J., \& Preacher, K. J. (2009). The hierarchical structure of wellbeing. Journal of Personality, 77, 1025-1049. doi:10.1111/j.1467-6494.2009.00573.x 
Gardner, R. M., Brown, D. L., \& Boice, R. (2012). Using Amazon’s Mechanical Turk website to measure accuracy of body size estimation and body dissatisfaction. Body Image, 9, 532-534. doi:10.1016/j.bodyim.2012.06.006

Gillen, M. M. (2015). Associations between positive body image and indicators of men's and women's mental and physical health. Body Image, 13, 67-74. doi:10.1016/j.bodyim.2015.01.002

Hair, J. F. Jr., Anderson, R. E., Tatham, R. L. \& Black, W. C. (1995). Multivariate data analysis ( $3^{\text {rd }}$ ed.). New York, NY: Macmillan.

Halliwell, E. (2015). Future directions for positive body image research. Body Image, 14, 177-189. doi:10.1016/j.bodyim.2015.03.003

Homan, K. J., \& Tylka, T. L. (2014). Appearance-based exercise motivation moderates the relationship between exercise frequency and positive body image. Body Image, 11, 101-108. doi:10.1016/j.bodyim.2014.01.003

Keyes, C. L. M. (1998). Social well-being. Social Psychology Quarterly, 61, 121-140.

Keyes, C. L. M. (2002). The mental health continuum: From languishing to flourishing in life. Journal of Health and Social Behavior, 43, 207-222.

Keyes, C. L. M. (2005). Mental illness and/or mental health? Investigating axions of the complete state model of health. Journal of Consulting and Clinical Psychology, 73, 539-548. doi:10.1037/0022-0006X.73.3.539

Keyes, C. L. M. (2006). Mental health in adolescence: Is America's youth flourishing? American Journal of Orthopsychiatry, 76, 395-402. doi:10.1037/0002-9432.76.3.395

Keyes, C. L. M., Shmotkin, D., \& Ryff, C. D. (2002). Optimizing well-being: The empirical encounter of two traditions. Journal of Personality and Social Psychology, 82, 10071022. doi:10.1037/0022-3514.82.6.1007 
Keyes, C. L. M., Wissing, M., Potgieter, J. P., Temane, M., Kruger, A., \& van Rooy, S. (2008). Evaluation of the Mental Health Continuum Short Form (MHC-SF) in Setswana speaking South Africans. Clinical Psychology and Psychotherapy, 15, 181192. doi:10.1002/cpp.572

Lamers, S. M. A., Westerhof, G. J., Bohlmeijer, E. T., ten Klooster, P. M., \& Keyes, C. L. M. (2010). Evaluating the psychometric properties of the Mental Health Continuum-Short Form (MHC-SF). Journal of Clinical Psychology, 67, 99-110. doi:10.1002/jclp.2074

Marta-Simões, J., Ferreira, C., \& Mendes, A. L. (2016). Exploring the effect of external shame on body appreciation among Portuguese young adults: The role of selfcompassion. Eating Behaviors, 23, 174-179. doi:10.1016/j.eatbeh.2016.10.006

Mason, W., \& Suri, S. (2012). Conducting behavioral research on Amazon's Mechanical Turk. Behavior Research Methods, 44, 1-23. doi:10.3758/s13428-011-0124-6

Ng, S.-K., Barron, D., \& Swami, V. (2015). Factor structure and psychometric properties of the Body Appreciation Scale among adults in Hong Kong. Body Image, 13, 1-8. doi:10.1016/j.bodyim.2014.10.009

Petrillo, G., Capone, V., Caso, D., \& Keyes, C. L. M. (2015). The Mental Health ContinuumShort Form (MHC-SF) as a measure of well-being in the Italian context. Social Indicators Research, 121, 291-312. doi:10.1007/s11205-014-0629-3

Pila, E., Brunet, J., Crocker, P. R. E., Kowalski, K. C., \& Sabiston, C. M. (2016). Intrapersonal characteristics of body-related guilt, shame, pride, and envy in Canadian adults. Body Image, 16, 100-106. doi:10.1016/j.bodyim.2016.01.001

Raque-Bogdan, T. L., Piontkowski, S., Hui, K., Ziemer, K. S., \& Garriot, P. O. (2016). Selfcompassion as a mediator between attachment anxiety and body appreciation: An exploratory model. Body Image, 19, 28-36. doi:10.1016/j.bodyim.2016.08.001 
Ryff, C. D. (1989). Happiness is everything, or is it? Explorations on the meaning of psychological well-being. Journal of Personality and Social Psychology, 57, 10691081. doi:10.1037/0022-3514.57.6.1069

Ryff, C. D., \& Keyes, C. L. M. (1995). The structure of psychological well-being revisited. Journal of Personality and Social Psychology, 69, 719-727. doi:10.1037/00223514.69.4.719

Sandoz, E. K., Wilson, K. G., Merwin, R. M., \& Kellum, K. K. (2013). Assessment of body image flexibility: The Body Image-Acceptance and Action Questionnaire. Journal of Contextual and Behavioral Science, 2, 39-48. doi:10.1016/ j.jcbs.2013.03.002

Spencer, E. A., Appleby, P. N., Davey, G. K., \& Key, T. J. (2002). Validity of self-reported height and weight in 4808 EPIC-Oxford participants. Public Health Nutrition, 5, 561565. doi:10.1079/PHN2001322

Sullivan, P. W., Ghushchvan, Wyatt, H. R., Wu, E. Q., \& Hill, J. O. (2007). Impact of cardiometabolic risk factor clusters on health-related quality of the life in the U.S. Obesity, 15, 511-521. doi:10.1038/oby.2007.580

Swami, V., Airs, N., Chouhan, B., Padilla Leon, M. A., \& Towell, T. (2009). Are there ethnic differences in positive body image among female British undergraduates? European Psychologist, 14, 288-296. doi:10.1027/1016-9040.14.4.288

Swami, V.,Barron, D., Weis, L., \& Furnham, A. (2016). Bodies in nature: Associations between exposure to nature, connectedness to nature, and body image. Body Image, 18, 153-161. doi:10.1016/j.bodyim.2016.07.002

Swami, V., Hadji-Michael, M., \& Furnham, A. (2008). Personality and individual difference correlates of positive body image. Body Image, 5, 322-325. doi:10.1016/j.bodyim.2008.03.007 
Swami, V., Henry, A., Peacock, N., Roberts-Dunn, A., \& Porter, A. (2013). "Mirror, mirror..." A preliminary investigation of skin tone dissatisfaction and its impact among British adults. Cultural Diversity and Ethnic Minority Psychology, 19, 468-476. doi:10.1037/a0032904

Swami, V., \& Ng, S.-K. (2015). Factor structure and psychometric properties of the Body Appreciation Scale-2 among adults in Hong Kong. Body Image, 15, 68-71. doi:10.1016/j.bodyim.2014.10.009

Swami, V., Ng, S.-K., \& Barron, D. (2016). Translation and psychometric evaluation of a Standard Chinese version of the Body Appreciation Scale-2. Body Image, 18, 23-26. doi:10.1016/j.bodyim.2016.04.005

Swami, V., Stieger, S., Haubner, T., \& Voracek, M. (2008). German translation and psychometric evaluation of Body Appreciation Scale. Body Image, 5, 122-127. doi:10.1016/j.bodyim.2007.10.002

Swami, V., Tran, U. S., Stieger, S., Voracek, M., \& The YouBeauty.com Team (2015). Associations between women's body image and happiness: Results of the YouBeauty.com Body Image Survey (YBIS). Journal of Happiness Studies, 16, 705716. doi:10.1007/s10902-014-9530-7

Swami, V., von Nordheim, L., \& Barron, D. (2016). Self-esteem mediates the relationship between connectedness to nature and body appreciation in women, but not men. Body Image, 16, 41-44. doi:10.1016/j.bodyim.2015.11.001

Tiggemann, M. (2015). Considerations of positive body image across various social identities and special populations. Body Image, 14, 168-176. doi:10.1016/j.bodyim.2015.03.002

Tiggemann, M., \& McCourt, A. (2013). Body appreciation in adult women: Relationships with age and body satisfaction. Body Image, 10, 624-627.

doi:10.1016/j.bodyim.2013.07.003 
Tylka, T. L. (2011). Positive psychology perspectives on body image. In T. F. Cash, \& L. Smolak (Eds.), Body image: A handbook of science, practice and prevention $\left(2^{\text {nd }} \mathrm{ed}\right.$., pp. 56-64). New York, NY: Guilford Press.

Tylka, T. L. (2012). Positive psychology perspectives on body image. In T. F. Cash (Ed.), Encyclopedia of body image and human appearance (Vol. 2, pp. 657-663). San Diego, CA: Academic Press.

Tylka, T. K., \& Iannantuono, A. C. (2016). Perceiving beauty in all women: Psychometric evaluation of the Broad Conceptualization of Beauty Scale. Body Image, 17, 67-81. doi:10.1016/j.bodyim.2016.02.005

Tylka, T. K., \& Kroon van Diest, A. M. (2013). The Intuitive Eating Scale-2: Item refinement and psychometric evaluation with college women and men. Journal of Counseling Psychology, 60, 137-153. doi:10.1037/a0030893

Tylka, T. L., \& Wood-Barcalow, N. L. (2015a). What is and what is not positive body image? Conceptual foundations and construct definition. Body Image, 14, 118-129. doi:10.1016/j.bodyim.2015.04.001

Tylka, T. L., \& Wood-Barcalow, N. L. (2015b). The Body Appreciation Scale-2: Item refinement and psychometric evaluation. Body Image, 12, 53-67. doi:10.1016/j.bodyim.2014.09.006

Wasylkiw, L., MacKinnon, A. L., \& MacLellan, S. A. (2014). Considering an affect regulation framework for examining the association between body dissatisfaction and positive body image in Black older adolescent females: Does body mass index matter? Body Image, 11, 426-437. doi:10.1016/j.bodyim.2014.07.002

Webb, J. B., Butler-Ajibade, P., \& Robinson, S. A. (2014). Considering an affect regulation framework for examining the association between body dissatisfaction and positive 
body image in Black older adolescent females: Does body mass index matter? Body Image, 11, 426-437. doi:10.1016/j.bodyim.2014.07. 002

Webb, J. B., Wood-Barcalow, N. L., \& Tylka, T. L. (2015). Assessing positive body image: Contemporary approaches and future directions. Body Image, 14, 130-145. doi:10.1016/j.bodyim.2015.03.010

Westerhof, G. J., \& Keyes, C. L. M. (2010). Mental illness and mental health: The two continua model across the lifespan. Journal of Adult Development, 17, 110-119. doi:10.1007/s10804-009-9082-y

Williams, E. P., Mesidor, M., Winters, K., Dubbert, P. M., \& Wyatt, S. B. (2015). Overweight and obesity: Prevalence, consequences, and causes of a growing public health problem. Current Obesity Reports, 4, 363-370. doi:10.1007/s13679-015-0169-4

Wood-Barcalow, N. L., Tylka, T. L., \& Augustus-Horvath, C. L. (2010). "But I like my body": Positive body image characteristics and a holistic model for young-adult women. Body Image, 7, 106-116. doi:10.1016/j.bodyim.2010.01.001 
Table 1. Descriptive Statistics and the Results of Between-Group Comparisons as a Function of Respondent Sex

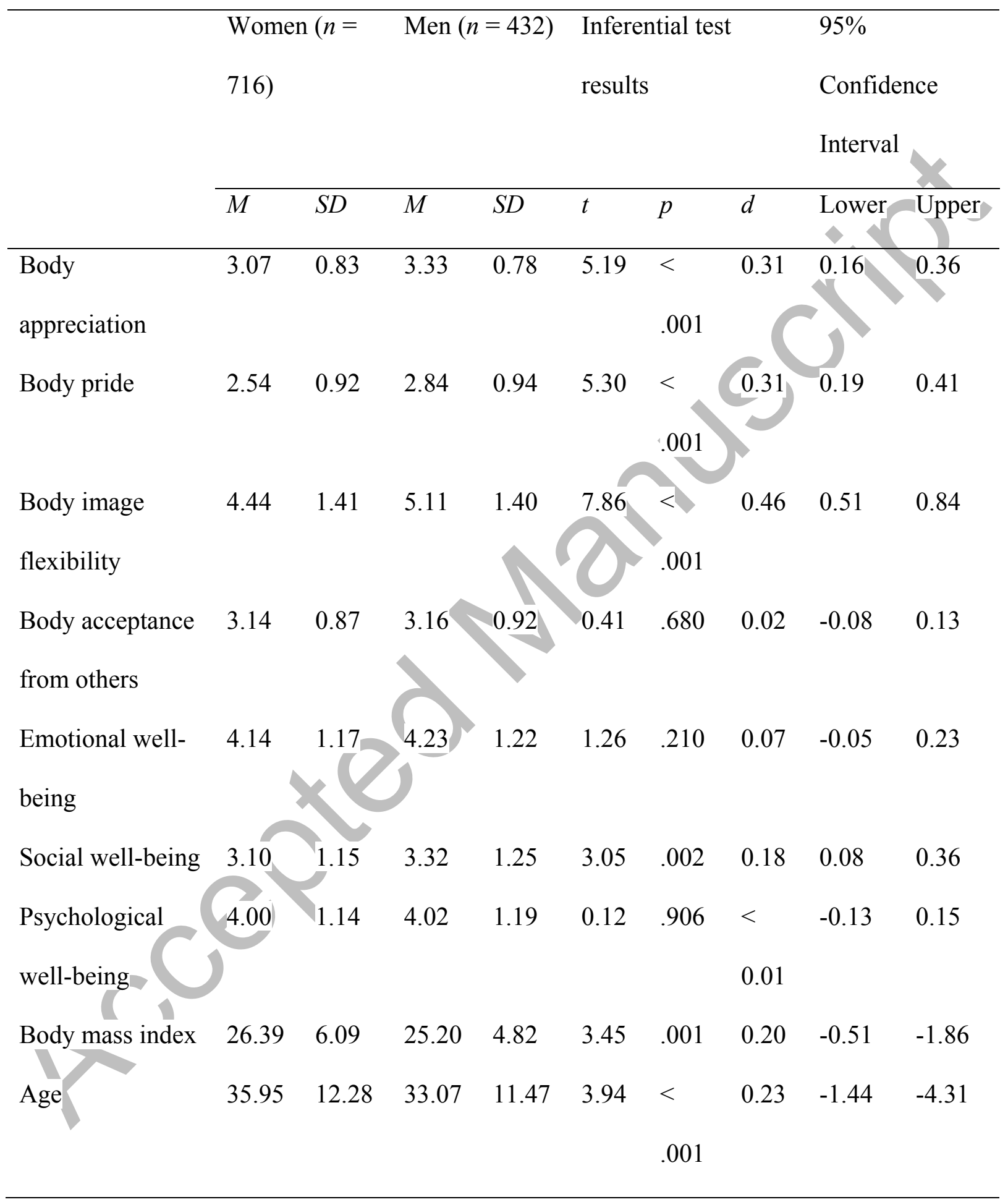

Note: Inferential tests were Bonferroni-corrected, such that $p$ was significant at $<.006$. 
Table 2. Inter-Scale Correlations between All Variables with Results for Women in the Top Diagonal and for Men in the Bottom Diagonal
(1)
(2)
(3)
(4)
(5)
(6)
(7)
(8)
(9)

(1) Body

$\begin{array}{lllllll}.65 * * & .71 * * & .53 * * & .60 * * & .59 * * & .49 * * & .01\end{array}$

appreciation

(2) Body image $\quad .30 * *$

$.44 * * \quad .43 * * \quad .47 * \quad .41 * * \quad .34 * * \quad .12 *$

flexibility

(3) Body pride $\quad .66^{* *} \quad .07$

$\begin{array}{lllll}.47 * & .43 * * \quad .46^{* *} \quad .40^{* *} & -.07\end{array}$

$.35^{* *}$

(4) Body

$.49^{* *} \quad .17 * * \quad .43^{* *}$

$.34 * * \quad .36 * * .26 * * \quad-.12 * \quad-$

acceptance from

$.37 * *$

others

(5) Emotional

$\begin{array}{llll}.63 * * & .27 * * & .44 * * & .37 * *\end{array}$

$.73 * * \quad .63 * * \quad .04$

well-being

(6) Psychological

$.61 * * \quad .16 *$

$.45 * * \quad .38 * * \quad .77 * *$

$\begin{array}{lll}.70 * * & .11 * & -.06\end{array}$

well-being

(7) Social well-

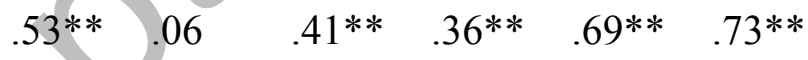

$.10^{*} \quad-.07$

being

(8) Age

$-.04$

$.14 *$

$-.09$

$-.14 * \quad .03$

.08

.01

$.16^{* *}$

(9) Body mass

$\begin{array}{lllll}-.15 * & -.04 & -.03 & -.01 & .19 * *\end{array}$

index

$.23^{* *} \quad .26^{* *} \quad .18^{* *}$

Note. Women $n=716$, men $n=432 .{ }^{*} p<.05,{ }^{* *} p<.001$. 
Table 3. Results of the Regression Analyses for Women $(n=716)$

\begin{tabular}{|c|c|c|c|c|c|c|c|c|c|c|}
\hline & \multicolumn{5}{|c|}{ Emotional well-being } & \multicolumn{5}{|c|}{ Psychological well-being } \\
\hline & $\mathrm{B}$ & $S E$ & $\beta$ & $t$ & $p$ & $\mathrm{~B}$ & $S E$ & $\beta$ & $t$ & $p$ \\
\hline Body appreciation & .72 & .07 & .50 & 9.99 & $<.001$ & .66 & .07 & .48 & 9.61 & $<.001$ \\
\hline Body image flexibility & .13 & .03 & .16 & 3.88 & $<.001$ & .06 & .03 & & 1.84 & .066 \\
\hline Body pride & .04 & .04 & .03 & 0.75 & .451 & .16 & & & 3.00 & .003 \\
\hline Body acceptance from others & .05 & .05 & .04 & 0.95 & .342 & .13 & & & 2.66 & .008 \\
\hline Age & .01 & .01 & .01 & 0.03 & .978 & & .0 & .09 & 2.82 & .005 \\
\hline Body mass index & -.02 & .01 & -.12 & -3.51 & $<.001$ & & .01 & -.20 & -6.05 & $<.001$ \\
\hline
\end{tabular}


Table 4. Results of the Regression Analyses for Men $(n=432)$

\begin{tabular}{|c|c|c|c|c|c|c|c|c|c|c|}
\hline & \multicolumn{5}{|c|}{ Emotional well-being } & \multicolumn{5}{|c|}{ Psychological well-being } \\
\hline & $\mathrm{B}$ & $S E$ & $\beta$ & $t$ & $p$ & B & $S E$ & $\beta$ & $t$ & $p$ \\
\hline Body appreciation & .82 & .08 & .53 & 9.86 & $<.001$ & .79 & .08 & .52 & 9.61 & $<.001$ \\
\hline Body image flexibility & .11 & .04 & .13 & 3.09 & .002 & .01 & .04 & .01 & 0.23 & .822 \\
\hline Body pride & .09 & .07 & .07 & 1.29 & .198 & .10 & .07 & & 1.47 & .142 \\
\hline Body acceptance from others & .12 & .06 & .09 & 2.01 & .045 & .17 & & & 2.92 & .004 \\
\hline Age & .01 & .01 & .03 & 0.86 & .391 & & .0 & .11 & 2.84 & .005 \\
\hline Body mass index & -.03 & .01 & -.13 & -3.26 & .001 & & .01 & -.10 & -2.58 & .010 \\
\hline
\end{tabular}

